\title{
Approximate Analytical Solution for Nonlinear System of Fractional Differential Equations by BPs Operational Matrices
}

\author{
Mohsen Alipour ${ }^{1}$ and Dumitru Baleanu ${ }^{2,3,4}$ \\ ${ }^{1}$ Department of Mathematics, Imam Khomeini International University, P.O. Box 34149-16818, Qazvin, Iran \\ ${ }^{2}$ Department of Mathematics and Computer Sciences, Cankaya University, 06530 Ankara, Turkey \\ ${ }^{3}$ Institute of Space Sciences, P.O. Box MG-23, 077125 Magurele-Bucharest, Romania \\ ${ }^{4}$ Department of Chemical and Materials Engineering, Faculty of Engineering, King Abdulaziz University, \\ P.O. Box 80204, Jeddah 21589, Saudi Arabia
}

Correspondence should be addressed to Mohsen Alipour; m.alipour2323@gmail.com

Received 20 February 2013; Revised 6 March 2013; Accepted 7 March 2013

Academic Editor: José Tenreiro Machado

Copyright (c) 2013 M. Alipour and D. Baleanu. This is an open access article distributed under the Creative Commons Attribution License, which permits unrestricted use, distribution, and reproduction in any medium, provided the original work is properly cited.

\begin{abstract}
We present two methods for solving a nonlinear system of fractional differential equations within Caputo derivative. Firstly, we derive operational matrices for Caputo fractional derivative and for Riemann-Liouville fractional integral by using the Bernstein polynomials (BPs). In the first method, we use the operational matrix of Caputo fractional derivative (OMCFD), and in the second one, we apply the operational matrix of Riemann-Liouville fractional integral (OMRLFI). The obtained results are in good agreement with each other as well as with the analytical solutions. We show that the solutions approach to classical solutions as the order of the fractional derivatives approaches 1.
\end{abstract}

\section{Introduction}

Differential equations of fractional order have been subjected to many studies due to their frequent appearance in various applications in fluid mechanics, viscoelasticity, biology, physics, engineering, and so on. Recently, a large amount of literature was developed regarding the application of fractional differential equations in nonlinear dynamics (see, e.g., [1-11] and the references therein). Thus, a huge attention has been given to the solution of fractional ordinary differential equations, integral equations, and fractional partial differential equations of physical interest. As it is known, there exists no method that yields an exact solution for fractional differential equations. Various methods have been proposed in order to solve the fractional differential equations. These methods include the homotopy perturbation method [1215], Adomian's decomposition method [16-20], variation iteration method [12-14, 21-23], homotopy analysis method [24], differential transform method [25], operational matrices [26-28], and nonstandard finite difference scheme [29].
In this paper, we investigate the nonlinear system of fractional differential equations as

$$
\begin{array}{r}
D^{\alpha_{i}} x_{i}(t)=g_{i}(t, X(t)), \\
i=1, \ldots, n, 0<t \leq 1,0<\alpha_{i} \leq 1,
\end{array}
$$

and the initial condition

$$
X(0)=X_{0}
$$

where $X(t)=\left[x_{1}(t), \ldots, x_{n}(t)\right]^{T}$ and $X_{0}=\left[x_{0,1}, \ldots, x_{0, n}\right]^{T}$. Also, $g_{i}:[0,1] \times R^{n} \rightarrow R$ are multivariable polynomial functions.

The structure of the paper is given later. In Section 2, we present some preliminaries and properties in fractional calculus and Bernstein polynomials. In Section 3, we make operational matrices for product, power, Caputo fractional derivative, and Riemann-Liouville fractional integral by BPs. In Section 4, we apply two methods for solving nonlinear 


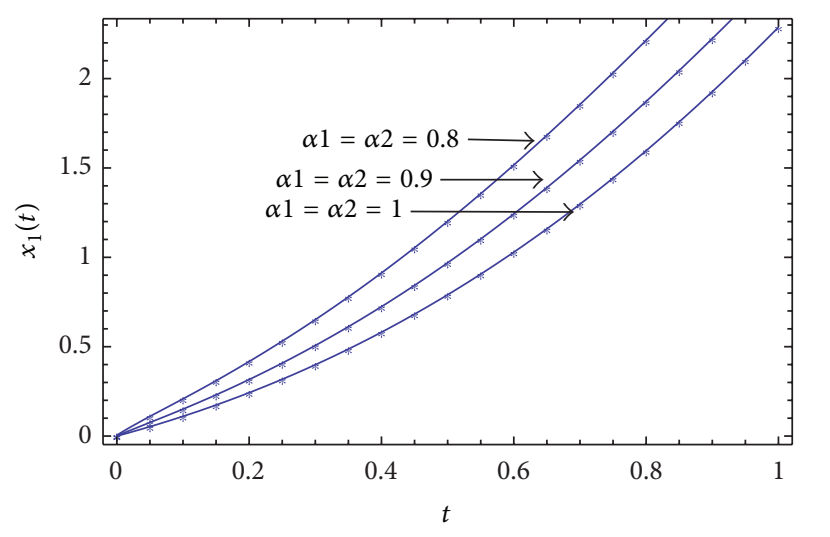

* OMCFD

- OMRLFI

FIgURE 1: Approximate solutions of $x_{1}(t)$ for $m=10$ and different values of $\alpha_{1}, \alpha_{2}$ by OMCFD and OMRLFI in Example 12.

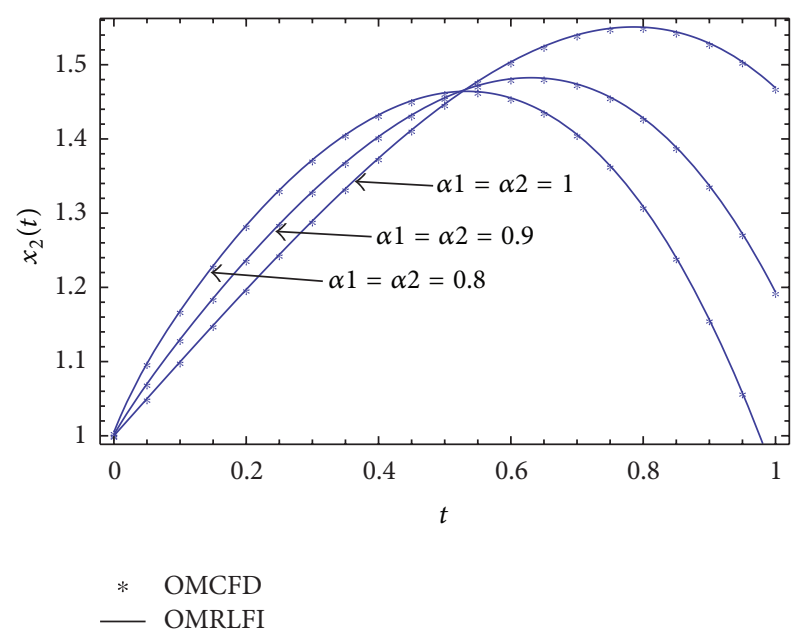

FIGURE 2: Approximate solutions of $x_{2}(t)$ for $m=10$ and different values of $\alpha_{1}, \alpha_{2}$ by OMCFD and OMRLFI in Example 12.

system of fractional differential equations by BPs. In Section 5, numerical examples are simulated to demonstrate the high performance of the proposed method. Conclusions are presented in Section 6.

\section{Basic Tools}

In this section, we recall some basic definitions and properties of the fractional calculus and Bernstein polynomials.

Definition 1 (see $[2,7,10]$ ). The Riemann-Liouville fractional integral operator of order $\alpha \geq 0$, of a function $f \in C_{\mu}, \mu \geq$ -1 , is defined as

$$
\begin{gathered}
I^{\alpha} f(t)=\frac{1}{\Gamma(\alpha)} \int_{0}^{t}(t-x)^{\alpha-1} f(x) d x, \quad \alpha>0, t>0, \\
I^{0} f(t)=f(t),
\end{gathered}
$$

and for $n-1<\alpha \leq n, n \in N, t>0, f \in C_{-1}^{n}$, the fractional derivative of $f(t)$ in the Caputo sense is defined as

$$
D^{\alpha} f(t)=\frac{1}{\Gamma(n-\alpha)} \int_{0}^{t}(t-x)^{n-\alpha-1} f^{(n)}(x) d x,
$$

where for $n \in N, \mu \in R$ we have

$$
\begin{gathered}
C_{\mu}=\{f(t) \mid f(t)>0 \text { for } t>0, \\
\left.f(t)=t^{p} f_{1}(t) \text { where } p>\mu, f_{1}(t) \in C[0, \infty)\right\}, \\
C_{\mu}^{n}=\left\{f(t) \mid f^{(n)}(t) \in C_{\mu}\right\} .
\end{gathered}
$$

Also, if $n-1<\alpha \leq n, n \in N$, and $f \in C_{\mu}^{n}, \mu \geq-1$, then

$$
\text { (1) } D^{\alpha} I^{\alpha} f(t)=f(t) \text {, }
$$

(2) $I^{\alpha} D^{\alpha} f(t)=f(t)-\sum_{k=0}^{n-1} f^{(k)}\left(0^{+}\right) \frac{x^{k}}{k !}, \quad t>0$.

Definition 2 (see [30]). The Bernstein polynomials (BPs) of $m$ th degree are defined on the interval $[0,1]$ as follows:

$$
B_{i, m}(x)=\left(\begin{array}{c}
m \\
i
\end{array}\right) x^{i}(1-x)^{m-i}, \quad i=0,1, \ldots, m .
$$

Lemma 3. One can write $\Phi_{m}(x)=A T_{m}(x)$, where $A$ is a matrix upper triangular, $T_{m}(x)=\left[1, x, \ldots, x^{m}\right]^{T}$, and $\Phi_{m}(x)=\left[B_{0, m}(x), B_{1, m}(x), \ldots, B_{m, m}(x)\right]^{T}$.

Proof. (see [26]).

Definition 4. We can define the dual matrix $Q_{(m+1) \times(m+1)}$ on the basis of Bernstein polynomials of $m$ th degree as follows:

$$
Q=\int_{0}^{1} \Phi(x) \Phi(x)^{T} d x
$$

where

$$
\begin{aligned}
(Q)_{i+1, j+1} & =\int_{0}^{1} B_{i, m}(x) B_{j, m}(x) d x \\
& =\frac{\left(\begin{array}{c}
m \\
i
\end{array}\right)\left(\begin{array}{c}
m \\
j
\end{array}\right)}{(2 m+1)\left(\begin{array}{c}
2 m \\
i+j
\end{array}\right)}, \quad i, j=0,1, \ldots, m .
\end{aligned}
$$

Lemma 5. Let $L^{2}[0,1]$ be a Hilbert space with the inner product $\langle f, g\rangle=\int_{0}^{1} f(x) g(x) d x$ and $y \in L^{2}[0,1]$. Then, we can find the unique vector $c=\left[c_{0}, c_{1}, \ldots, c_{m}\right]^{T}$ such that $c^{T} \Phi_{m}(x)$ is the best approximation of $y(x)$ from space $S_{m}=\operatorname{Span}\left\{B_{0, m}(x), B_{1, m}(x), \ldots, B_{m, m}(x)\right\}$. Moreover, one can get $c=Q^{-1}\left\langle y, \Phi_{m}\right\rangle$, such that $\left\langle y, \Phi_{m}\right\rangle=\left[\left\langle y, B_{0, m}\right\rangle\right.$, $\left.\left\langle y, B_{1, m}\right\rangle, \ldots,\left\langle y, B_{m, m}\right\rangle\right]^{T}$.

Proof. (see [31]). 


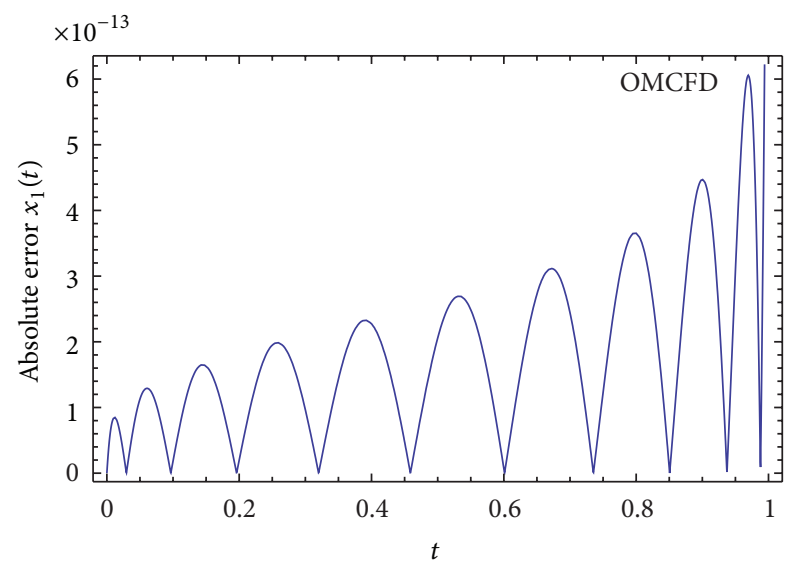

(a)

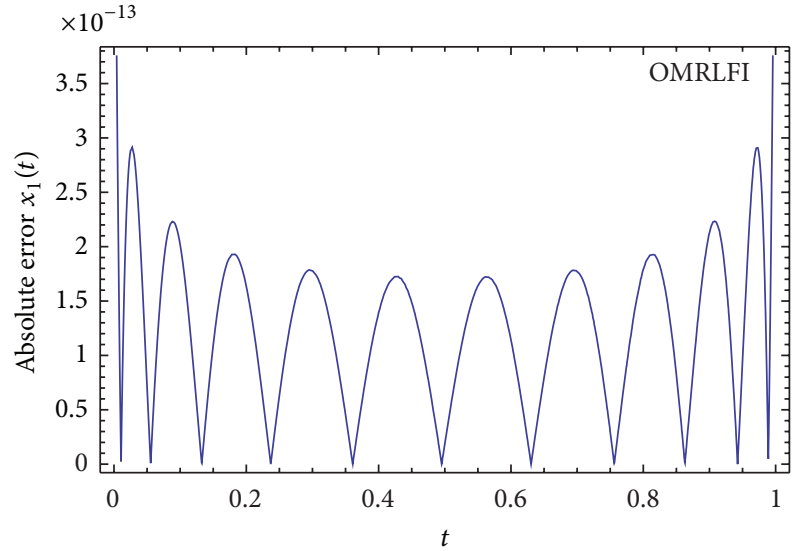

(b)

FIgURE 3: Plot of absolute error function $x_{1}(t)$ for $\alpha_{1}=\alpha_{2}=1$ and $m=10$ by OMCFD and OMRLFI in Example 12.

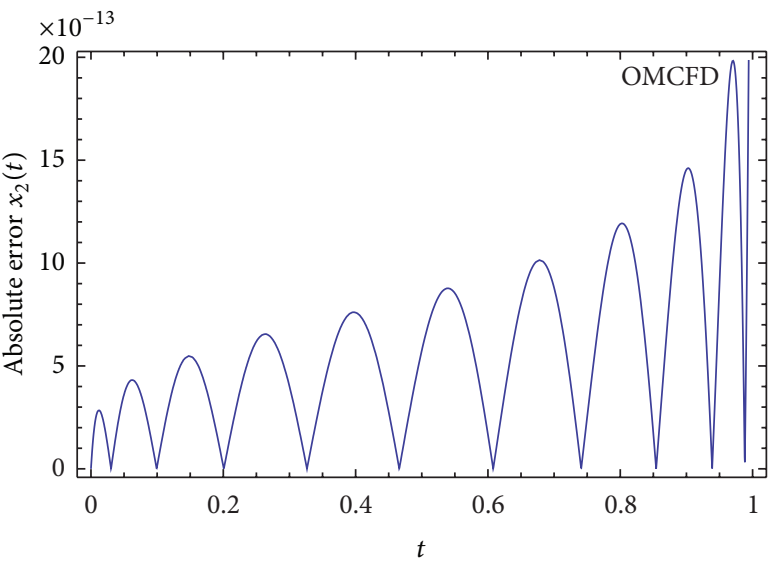

(a)

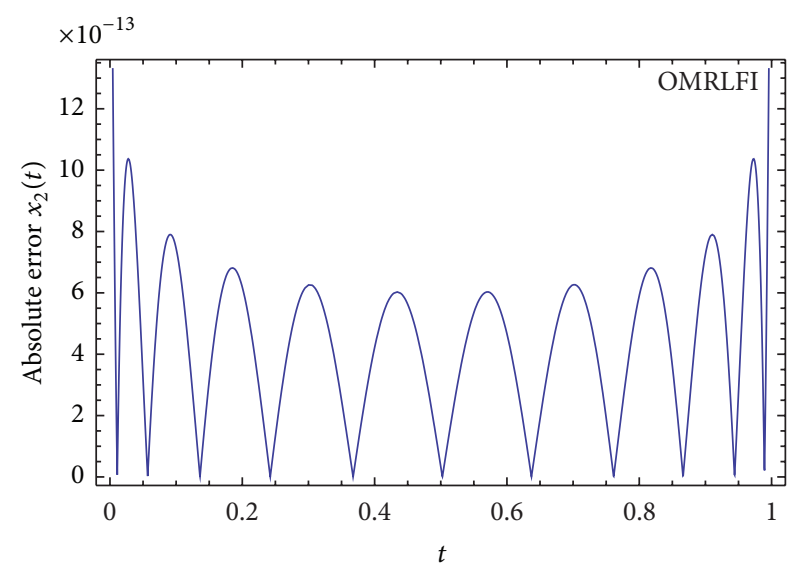

(b)

FIGURE 4: Plot of absolute error function $x_{2}(t)$ for $\alpha_{1}=\alpha_{2}=1$ and $m=10$ by OMCFD and OMRLFI in Example 12.

Lemma 6. Suppose that the function $y:[0,1] \rightarrow R$ is $m+1$ times continuously differentiable (i.e., $\left.y \in C^{m+1}([0,1])\right)$. If $c^{T} B$ is the best approximation $y$ out of $S_{m}$, then

$$
\left\|y-c^{T} B\right\|_{L^{2}[0,1]} \leq \frac{\widehat{K}}{(m+1) ! \sqrt{2 m+3}},
$$

where $\widehat{K}=\max _{x \in[0,1]}\left|y^{(m+1)}(x)\right|$. Also, if $y \in C^{\infty}([0,1])$, then the error bound vanishes.

Proof. (see [32]).

\section{Operational Matrices of Bernstein Polynomials}

In Section 3, we recall the operational matrices for product, power, Caputo fractional derivative and Riemann-Liouville fractional integral by BPs.
Lemma 7. Suppose that $c_{(m+1) \times 1}$ is an arbitrary vector. The operational matrix of product $\widehat{C}_{(m+1) \times(m+1)}$ using BPs can be given as follows:

$$
c^{T} \Phi_{m}(x) \Phi_{m}(x)^{T} \approx \Phi_{m}(x)^{T} \widehat{C} .
$$

Proof. (see [27]).

Corollary 8. Suppose that $y(t) \approx c^{T} \Phi_{m}(t), x(t) \approx d^{T} \Phi_{m}(t)$, and $\widehat{C}_{(m+1) \times(m+1)}$ is the operational matrix of product using $B P s$ for vector $c$. One can get the approximate function for $x(t) y(t)$ using BPs as follows:

$$
y(t) x(t) \approx \Phi_{m}(t)^{T} \widehat{C} d .
$$

Proof. By using Lemma 7, it is clear.

Corollary 9. Suppose that $y(t) \approx c^{T} \Phi_{m}(t)$ and $\widehat{C}_{(m+1) \times(m+1)}$ is the operational matrix of product using BPs for vector $c$. One 


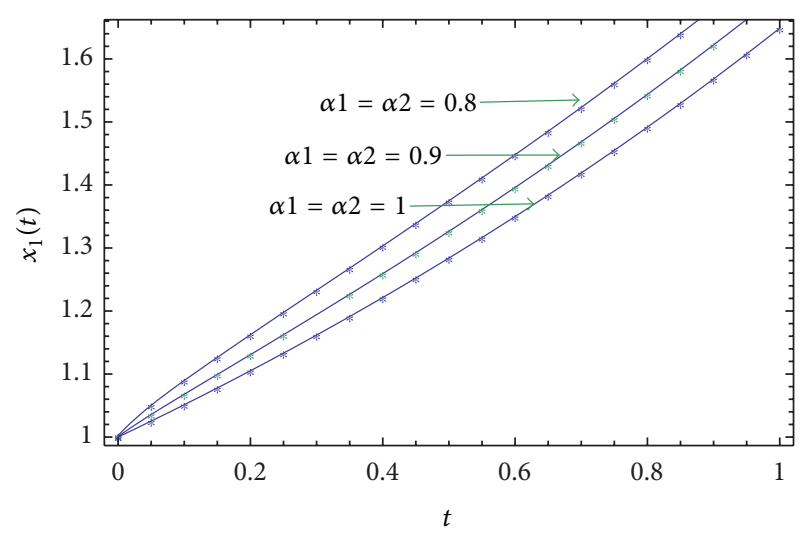

* OMCFD

- OMRLFI

FIGURE 5: Approximate solutions of $x_{1}(t)$ for $m=10$ and different values of $\alpha_{1}, \alpha_{2}$ by OMCFD and OMRLFI in Example 13.

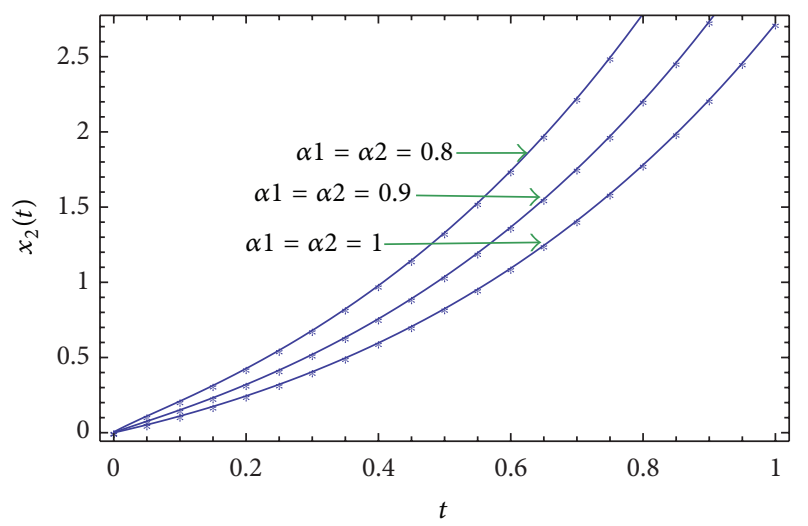

* OMCFD

— OMRLFI

Figure 6: Approximate solutions of $x_{2}(t)$ for $m=10$ and different values of $\alpha_{1}, \alpha_{2}$ by OMCFD and OMRLFI in Example 13.

can get the approximate function for $y^{k}(t)(k \in N)$, using BPs as follows:

$$
y^{k}(t) \approx \Phi_{m}(t)^{T} \widetilde{C}_{k}
$$

where $\widetilde{C}_{k}=\widehat{C}^{k-1} c$.

Proof. (see [26]).

Theorem 10. One can get BPs operational matrix $D_{\alpha}$ from order $(m+1) \times(m+1)$ for the Caputo fractional derivative as follows:

$$
\begin{aligned}
D^{\alpha} \Phi_{m}(t) & =\frac{1}{\Gamma(n-\alpha)} \int_{0}^{t}(t-x)^{n-\alpha-1} \Phi_{m}^{(n)}(x) d x \\
& \approx D_{\alpha} \Phi_{m}(t)
\end{aligned}
$$

Proof. See [26] for details.

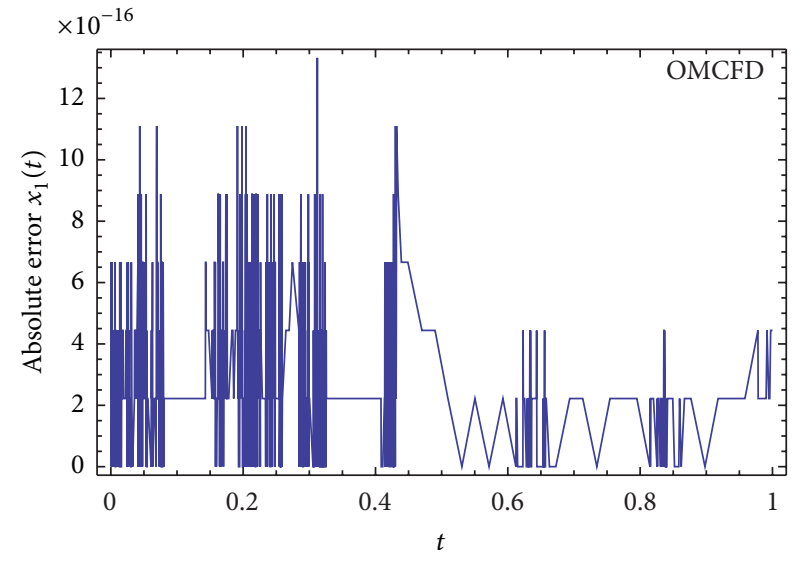

(a)

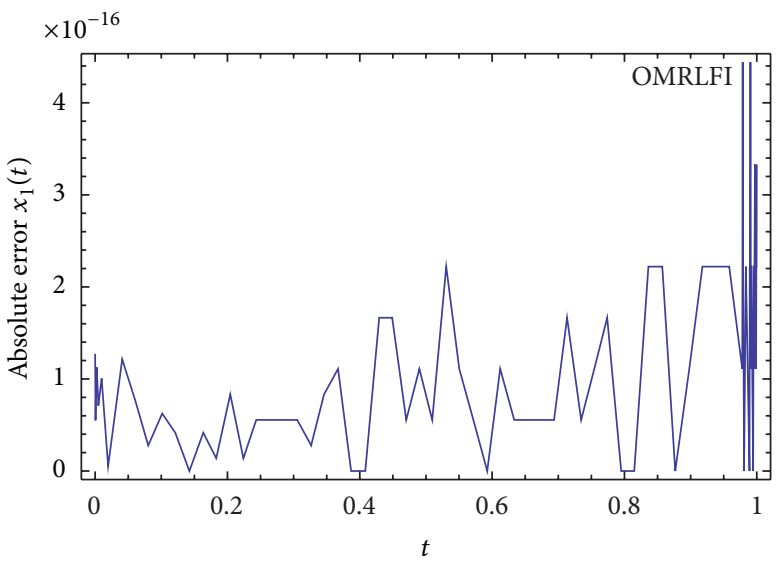

(b)

Figure 7: Plot of absolute error function $x_{1}(t)$ for $\alpha_{1}=\alpha_{2}=1$ and $m=10$ by OMCFD and OMRLFI in Example 13 .

Theorem 11. One can obtain the operational matrix $F_{\alpha}$ from order $(m+1) \times(m+1)$ for the Riemann-Liouville fractional integral on the basis of BPs from order $m$ as

$$
I^{\alpha} \Phi_{m}(t)=\frac{1}{\Gamma(\alpha)} \int_{0}^{t}(t-x)^{\alpha-1} \Phi_{m}(x) d x \approx F_{\alpha} \Phi_{m}(t) .
$$

Proof. See [28] for details.

\section{Solving System of Fractional Differential Equations}

In this section, we use two methods for solving system of fractional differential equations. In the first method, we use the operational matrix for Caputo fractional derivative (OMCFD), and in the second method, we apply the operational matrix for Riemann-Liouville fractional integral (OMRLFI).

4.1. Solving the Problem by OMCFD. Using Lemma 5, we can approximate the functions $x_{i}(t)$ as follows:

$$
x_{i}(t) \approx C_{i}^{T} \Phi_{m}(t), \quad i=1, \ldots, n,
$$

where $C_{i} \in R^{(m+1) \times 1}$. 


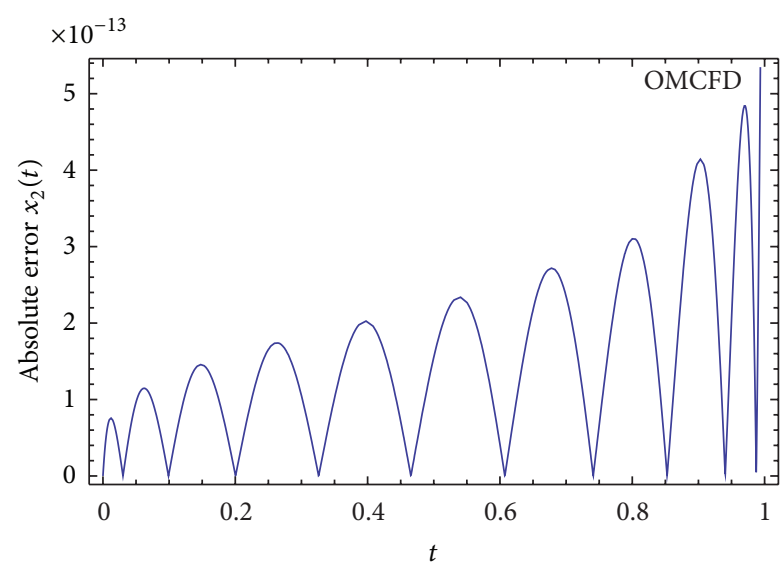

(a)

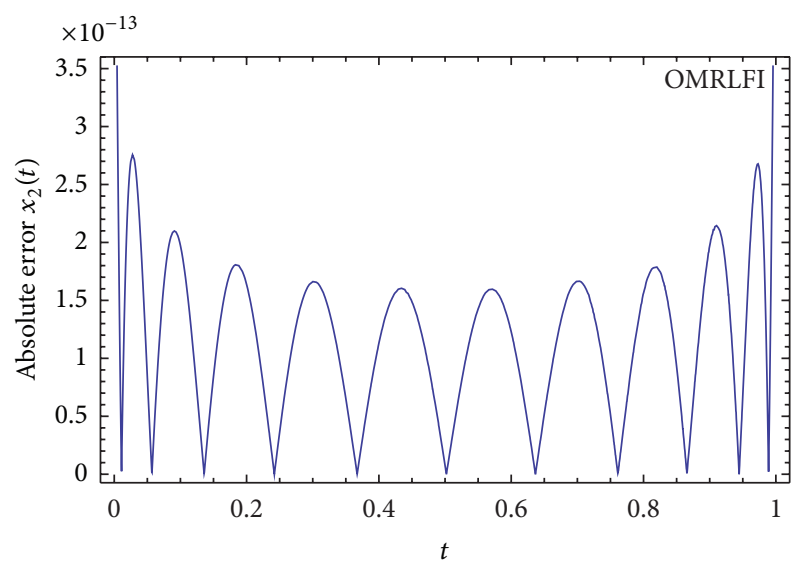

(b)

FIGURE 8: Plot of absolute error function $x_{2}(t)$ for $\alpha_{1}=\alpha_{2}=1$ and $m=10$ by OMCFD and OMRLFI in Example 13 .

From (17) and (15) we can write

$$
D^{\alpha_{i}} x_{i}(t) \approx C_{i}^{T} D_{\alpha_{i}} \Phi_{m}(t), \quad i=0, \ldots, n .
$$

Therefore, problem (1) and (2) reduces to the following problem:

$$
\begin{array}{r}
C_{i}^{T} D_{\alpha_{i}} \Phi_{m}(t)=g_{i}\left(t, C_{1}^{T} \Phi_{m}(t), \ldots, C_{n}^{T} \Phi_{m}(t)\right), \\
i=1, \ldots, n,
\end{array}
$$

and the initial condition

$$
C_{i}^{T} \Phi_{m}(0)=x_{0, i}, \quad i=1, \ldots, n
$$

Now, using Lemma 5 we can approximate all of the known functions in the system (19). Then, by using Lemma 7 and Corollaries 8 and 9 , since functions $g_{i}$ are polynomial, we obtain the following approximations:

$$
g_{i}(t, X(t)) \approx G_{i}\left(C_{1}, \ldots, C_{n}\right) \Phi_{m}(t), \quad i=1, \ldots, n,
$$

where $G_{i}: R^{(m+1) \times n} \rightarrow R^{1 \times(m+1)}$.
Also, for each $i(i=1, \ldots, n)$, by using tau method [33] we can generate algebraic equations from (19) and (21) as follows

$$
\begin{aligned}
\widetilde{G}_{i, j}=\int_{0}^{1}\left(C_{i}^{T} D_{\alpha_{i}}-G_{i}\left(C_{1}, \ldots, C_{n}\right)\right) \\
\quad \times \Phi_{m}(t) B_{j, m}(t) d t=0, \quad j=0, \ldots, m-1,
\end{aligned}
$$

and from (23) we set $\widetilde{G}_{i, m}=C_{i}^{T} \Phi_{m}(0)-x_{0, i}$.

Finally, problem (1) and (2) has been reduced to the system of algebraic equations

$$
\widetilde{G}_{i, j}\left(C_{1}, \ldots, C_{n}\right)=0, \quad i=1, \ldots, n, j=0, \ldots, m .
$$

The aforementioned system can be solved for $C_{i}$ by Newton's iterative method. Then, we get the approximate value of the functions $x_{i}(t)$ from (17).

4.2. Solving the Problem by OMRLFI. This method consists of two steps.

Step 1. Initial conditions are used to reduce a given initialvalue problem to a problem with zero initial conditions. Therefore we have a modified system, incorporating the initial values.

Step 2. The BPs operational matrix of Riemann-Liouville fractional integral is used to transform the problem into a system of algebraic equations.

Now, from (2) we define

$$
x_{i}(t)=x_{0, i}+z_{i}(t), \quad i=1,2, \ldots, n,
$$

where $z_{i}(t), i=1,2, \ldots, n$, are the new unknown functions.

Substituting (24) in (1) and (2), we have the following system:

$$
\begin{array}{r}
D^{\alpha_{i}} z_{i}(t)=f_{i}(t, Z(t)), \\
i=1, \ldots, n, 0<t \leq 1,0<\alpha_{i} \leq 1,
\end{array}
$$

and the initial condition

$$
z_{i}(0)=0, \quad i=1, \ldots, n
$$

where $Z(t)=\left[z_{1}(t), \ldots, z_{n}(t)\right]^{T}$ and $f_{i}:[0,1] \times R^{n} \rightarrow R$ are multivariable polynomial functions. We use the following approximation:

$$
D^{\alpha_{i}} z_{i}(t) \approx \widetilde{C}_{i}^{T} \Phi_{m}(t), \quad i=1, \ldots, n,
$$

where $\widetilde{C}_{i} \in R^{(m+1) \times 1}$ are unknown vectors. From (7), (27), and Theorem 11, we can write

$$
\begin{aligned}
z_{i}(t) & =I^{\alpha_{i}} D^{\alpha_{i}} z_{i}(t) \approx I^{\alpha_{i}}\left(\widetilde{C}_{i}^{T} \Phi_{m}(t)\right) \\
& =\widetilde{C}_{i}^{T} I^{\alpha_{i}} \Phi_{m}(t) \approx \widetilde{C}_{i}^{T} F_{\alpha_{i}} \Phi_{m}(t) .
\end{aligned}
$$




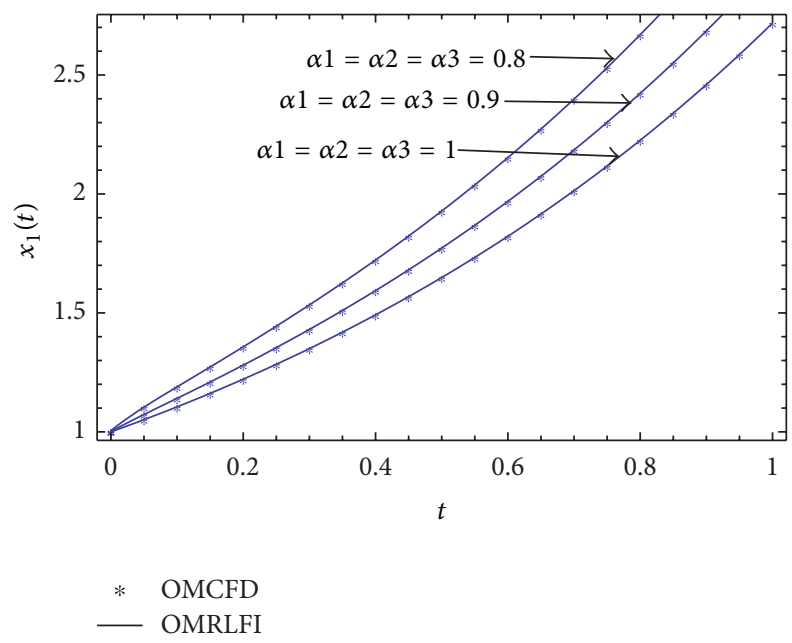

Figure 9: Approximate solutions of $x_{1}(t)$ for $m=10$ and different values of $\alpha_{1}, \alpha_{2}, \alpha_{3}$ by OMCFD and OMRLFI in Example 14.

So, by (27) and (28), problem (25) and (26) reduces to the following problem:

$$
\begin{array}{r}
\widetilde{C}_{i}^{T} \Phi_{m}(t)=f_{i}\left(t, \widetilde{C}_{1}^{T} F_{\alpha_{1}} \Phi_{m}(t), \ldots, \widetilde{C}_{n}^{T} F_{\alpha_{n}} \Phi_{m}(t)\right), \\
i=1, \ldots, n .
\end{array}
$$

As we saw in the previous section, we can obtain the following approximations:

$$
f_{i}(t, X(t)) \approx F_{i}\left(\widetilde{C}_{1}, \ldots, \widetilde{C}_{n}\right) \Phi_{m}(t), \quad i=1, \ldots, n,
$$

where $F_{i}: R^{(m+1) \times n} \rightarrow R^{1 \times(m+1)}$. So, from (29) and (30) we have

$$
\left(\widetilde{C}_{i}^{T}-F_{i}\left(\widetilde{C}_{1}, \ldots, \widetilde{C}_{n}\right)\right) \Phi_{m}(t)=0, \quad i=1, \ldots, n .
$$

Therefore, we have reduced problem (1) and (2) to the system of algebraic equations as follows:

$$
\widetilde{C}_{i}^{T}-F_{i}\left(\widetilde{C}_{1}, \ldots, \widetilde{C}_{n}\right)=0,
$$

where this system can be solved for $\widetilde{C}_{i}$ by Newton's iterative method. Finally we obtain the approximate of the functions $x_{i}(t)$ by

$$
x_{i}(t) \approx x_{0, i}+\widetilde{C}_{i}^{T} F_{\alpha_{i}} \Phi_{m}(t), \quad i=1,2, \ldots, n .
$$

\section{Examples}

To demonstrate the applicability and to validate the numerical scheme, we apply the present method for the following examples.

Example 12. Consider the following linear system of fractional differential equations $[24,25]$ :

$$
\begin{gathered}
D^{\alpha_{1}} x_{1}(t)=x_{1}(t)+x_{2}(t), \\
D^{\alpha_{2}} x_{2}(t)=-x_{1}(t)+x_{2}(t), \quad 0<\alpha_{1}, \alpha_{2} \leq 1,
\end{gathered}
$$

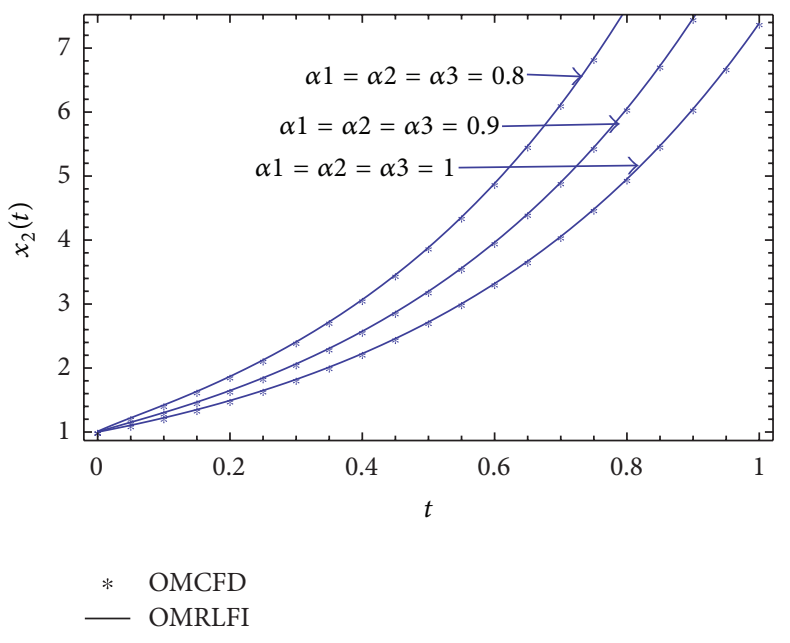

FIGURE 10: Approximate solutions of $x_{2}(t)$ for $m=10$ and different values of $\alpha_{1}, \alpha_{2}, \alpha_{3}$ by OMCFD and OMRLFI in Example 14.

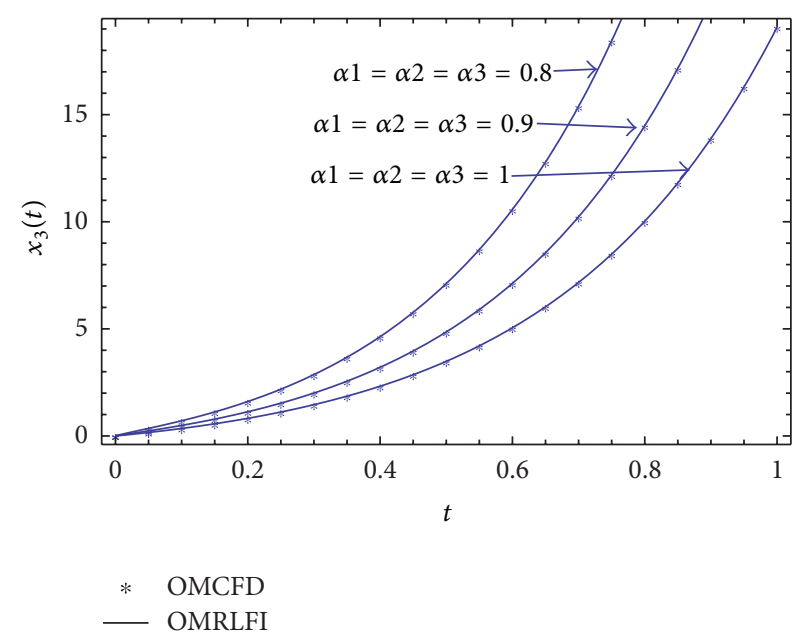

Figure 11: Approximate solutions of $x_{3}(t)$ for $m=10$ and different values of $\alpha_{1}, \alpha_{2}, \alpha_{3}$ by OMCFD and OMRLFI in Example 14.

with initial condition

$$
x_{1}(0)=0, \quad x_{2}(0)=1 .
$$

For this problem we have the exact solution in the case of $\alpha_{1}=$ $\alpha_{2}=1$ as

$$
\begin{aligned}
& x_{1}(t)=e^{t} \sin (t), \\
& x_{2}(t)=e^{t} \cos (t) .
\end{aligned}
$$

We solved this problem by OMCFD and OMRLFI. Figures 1 and 2 show the approximate solutions of $x_{1}(t)$ and $x_{2}(t)$, respectively, as a function of time for $m=10$, for different values of $\alpha_{1}, \alpha_{2}$. The results show that numerical solutions are in good agreement with each other, in both methods. Also, these figures show that as $\alpha_{1}, \alpha_{2}$ approach close to 1 , the numerical solutions approach to the solutions for $\alpha_{1}=\alpha_{2}=1$ as expected. In Figures 3 and 4, we see the absolute error of 


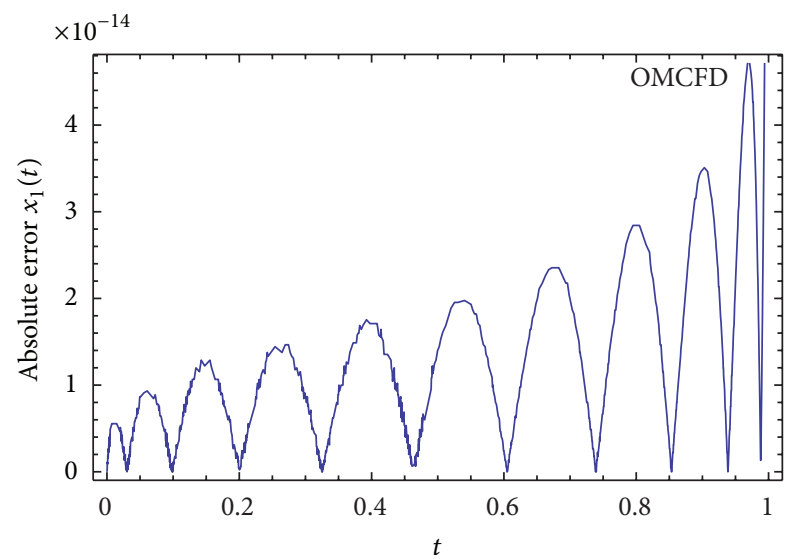

(a)

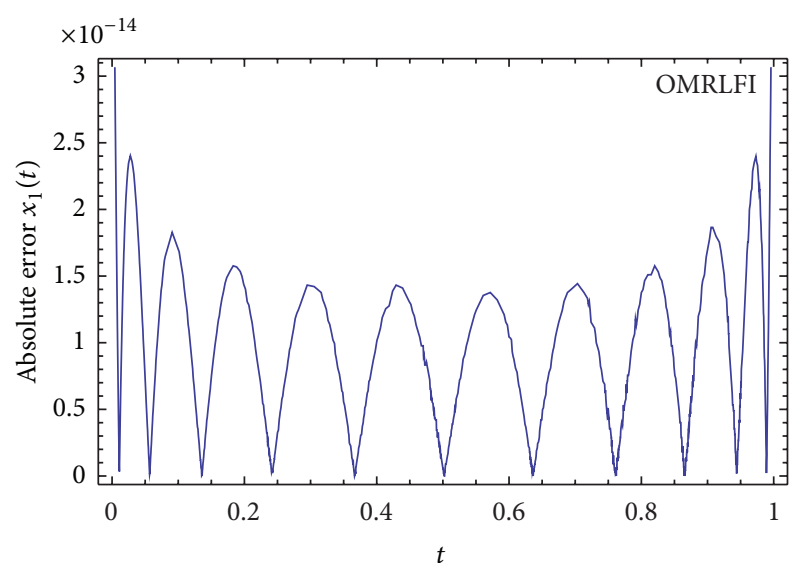

(b)

FIGURE 12: Plot of absolute error function $x_{1}(t)$ for $\alpha_{1}=\alpha_{2}=\alpha_{3}=1$ and $m=10$ by OMCFD and OMRLFI in Example 14 .

both methods, for $m=10, \alpha_{1}=\alpha_{2}=1$. In these figures, we can see that obtained results using the presented methods agree well with the analytical solutions for $\alpha_{1}=\alpha_{2}=1$.

Example 13. Let us consider the following nonlinear fractional system [24] as follows:

$$
\begin{gathered}
D^{\alpha_{1}} x_{1}(t)=\frac{x_{1}(t)}{2}, \\
D^{\alpha_{2}} x_{2}(t)=x_{1}^{2}(t)+x_{2}(t), \quad 0<\alpha_{1}, \alpha_{2} \leq 1,
\end{gathered}
$$

such that

$$
x_{1}(0)=1, \quad x_{2}(0)=0 .
$$

The exact solution of this system, when $\alpha_{1}=\alpha_{2}=1$, is

$$
\begin{aligned}
& x_{1}(t)=e^{t / 2} \\
& x_{2}(t)=t e^{t}
\end{aligned}
$$

Figures 5 and 6 show the approximate solutions of $x_{1}(t)$ and $x_{2}(t)$, respectively, for different values of $\alpha_{1}, \alpha_{2}$ by OMCFD

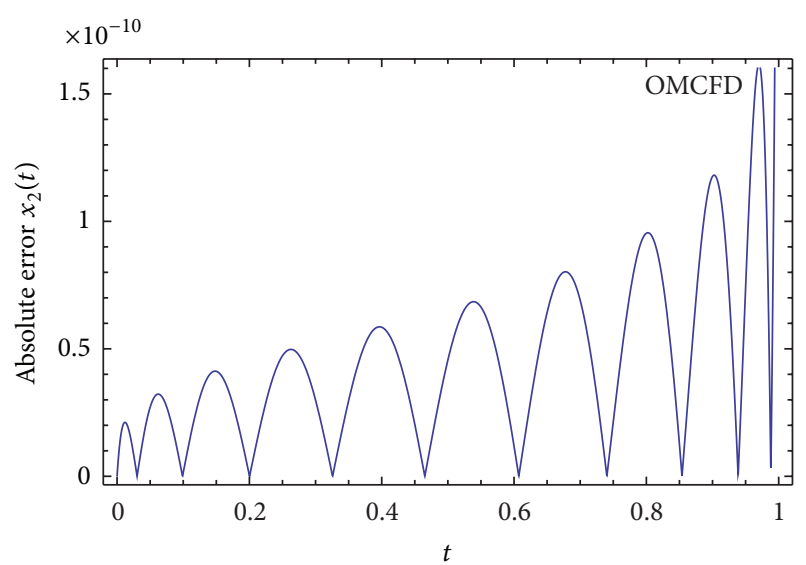

(a)

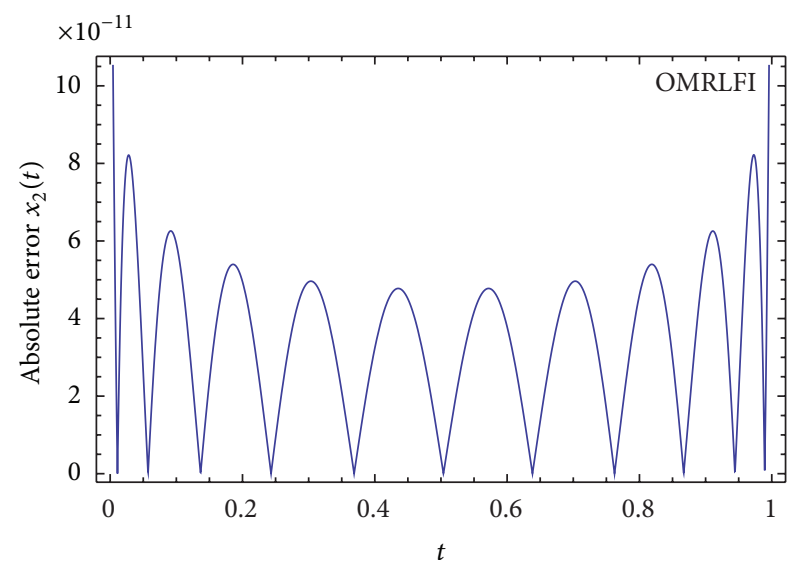

(b)

FIGURE 13: Plot of absolute error function $x_{2}(t)$ for $\alpha_{1}=\alpha_{2}=\alpha_{3}=1$ and $m=10$ by OMCFD and OMRLFI in Example 14 .

and OMRLFI. We conclude that as $\alpha_{1}, \alpha_{2}$ approach close to 1 , the numerical solutions approach solutions for $\alpha_{1}=\alpha_{2}=1$ as expected. Furthermore, in both methods, the results agree well with each other. Figures 7 and 8 show that, the absolute error of obtained results for $m=10$ and $\alpha_{1}=\alpha_{2}=1$ using OMCFD and OMRLFI is in good agreement with the exact solution.

Example 14. Consider the nonlinear system of fractional differential equations [24]:

$$
\begin{gathered}
D^{\alpha_{1}} x_{1}(t)=x_{1}(t), \\
D^{\alpha_{2}} x_{2}(t)=2 x_{1}^{2}(t), \\
D^{\alpha_{3}} x_{3}(t)=3 x_{1}(t) x_{2}(t), \quad 0<\alpha_{1}, \alpha_{2}, \alpha_{3} \leq 1,
\end{gathered}
$$

with the initial conditions given by

$$
x_{1}(0)=1, \quad x_{2}(0)=1, \quad x_{3}(0)=0 \text {. }
$$




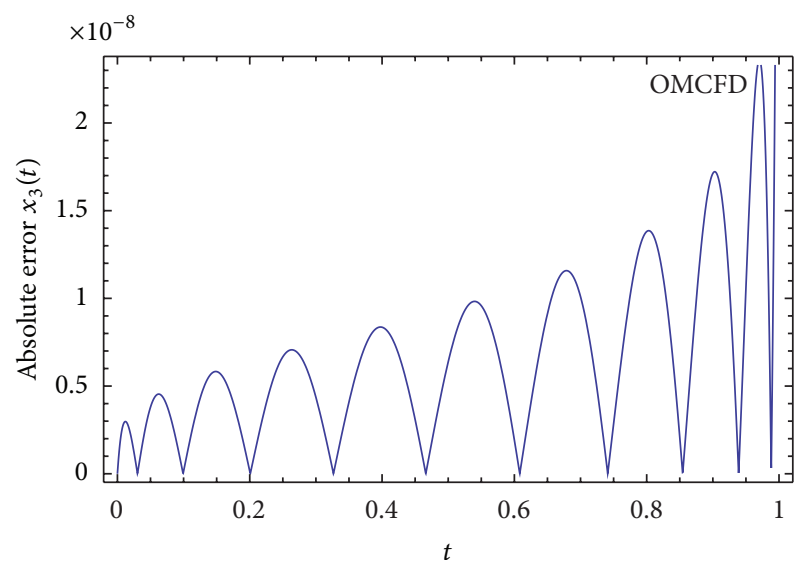

(a)

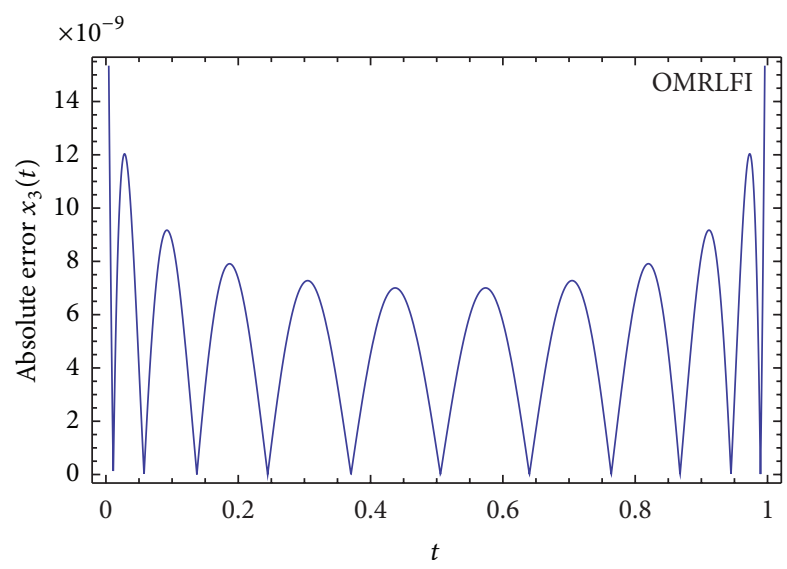

(b)

Figure 14: Plot of absolute error function $x_{3}(t)$ for $\alpha_{1}=\alpha_{2}=\alpha_{3}=1$ and $m=10$ by OMCFD and OMRLFI in Example 14 .

The exact solution of this system, when $\alpha_{1}=\alpha_{2}=\alpha_{3}=1$, becomes

$$
\begin{gathered}
x_{1}(t)=e^{t}, \\
x_{2}(t)=e^{2 t}, \\
x_{3}(t)=e^{3 t}-1 .
\end{gathered}
$$

We can see the approximate solutions of $x_{1}(t), x_{2}(t)$ and $x_{3}(t)$, by OMCFD and OMRLFI for $m=10$ and different values of $\alpha_{1}, \alpha_{2}$ and $\alpha_{3}$, in Figures 9, 10, and 11. These figures show that, when $\alpha_{1}, \alpha_{2}$, and $\alpha_{3}$ approach close to 1 , the numerical solutions approach the solutions for $\alpha_{1}=\alpha_{2}=$ $\alpha_{3}=1$ as expected. In Figures 9-11, we observe that results of OMCFD and OMRLFI overlap. In Figures 12, 13, and 14, we see the absolute error of the obtained results for $m=10$ and $\alpha_{1}=\alpha_{2}=\alpha_{3}=1$ in both methods.

\section{Conclusion}

In this paper, we get operational matrices of the product, Caputo fractional derivative, and Riemann-Liouville fractional integral by Bernstein polynomials. Then by using these matrices, we proposed two methods that reduced the nonlinear systems of fractional differential equations to the two system of algebraic equations that can be solved easily. Finally, numerical examples are simulated to demonstrate the high performance of the proposed method. We saw that the results of both methods were in good agreement with each other, and the classical solutions were recovered when the order of the fractional derivative goes to 1 .

\section{References}

[1] J.-H. He, "Approximate analytical solution for seepage flow with fractional derivatives in porous media," Computer Methods in Applied Mechanics and Engineering, vol. 167, no. 1-2, pp. 57-68, 1998.

[2] I. Podlubny, Fractional Differential Equations, Academic Press, New York, NY, USA, 1999.

[3] R. Hilfer, Applications of Fractional Calculus in Physics, World Scientific, Singapore, 2000.

[4] X. Gao and J. Yu, "Synchronization of two coupled fractionalorder chaotic oscillators," Chaos, Solitons and Fractals, vol. 26, no. 1, pp. 141-145, 2005.

[5] J. G. Lu, "Chaotic dynamics and synchronization of fractionalorder Arneodo's systems," Chaos, Solitons \& Fractals, vol. 26, no. 4, pp. 1125-1133, 2005.

[6] J. G. Lu and G. Chen, "A note on the fractional-order Chen system," Chaos, Solitons \& Fractals, vol. 27, no. 3, pp. 685-688, 2006.

[7] A. A. Kilbas, H. M. Srivastava, and J. J. Trujillo, Theory and Applications of Fractional Differential Equations, Elsevier, San Diego, Calif, USA, 2006.

[8] D. Baleanu, O. G. Mustafa, and R. P. Agarwal, "An existence result for a superlinear fractional differential equation," Applied Mathematics Letters, vol. 23, no. 9, pp. 1129-1132, 2010.

[9] D. Baleanu, O. G. Mustafa, and R. P. Agarwal, "On the solution set for a class of sequential fractional differential equations," Journal of Physics A, vol. 43, no. 38, Article ID 385209, 2010.

[10] D. Baleanu, K. Diethelm, E. Scalas, and J. J. Trujillo, Fractional Calculus Models and Numerical Methods, Series on Complexity, Nonlinearity and Chaos, World Scientific, Hackensack, NJ, USA, 2012.

[11] S. Bhalekar, V. Daftardar-Gejji, D. Baleanu, and R. L. Magin, "Transient chaos in fractional Bloch equations," Computers \& Mathematics with Applications, vol. 64, no. 10, pp. 3367-3376, 2012.

[12] S. Momani and Z. Odibat, "Numerical approach to differential equations of fractional order," Journal of Computational and Applied Mathematics, vol. 207, no. 1, pp. 96-110, 2007.

[13] S. Momani and Z. Odibat, "Homotopy perturbation method for nonlinear partial differential equations of fractional order," Physics Letters A, vol. 365, no. 5-6, pp. 345-350, 2007.

[14] S. Momani and Z. Odibat, "Numerical comparison of methods for solving linear differential equations of fractional order," Chaos, Solitons \& Fractals, vol. 31, no. 5, pp. 1248-1255, 2007.

[15] Z. Odibat and S. Momani, "Modified homotopy perturbation method: application to quadratic Riccati differential equation of fractional order," Chaos, Solitons \& Fractals, vol. 36, no. 1, pp. 167-174, 2008.

[16] S. Momani and K. Al-Khaled, "Numerical solutions for systems of fractional differential equations by the decomposition 
method," Applied Mathematics and Computation, vol. 162, no. 3, pp. 1351-1365, 2005.

[17] H. Jafari and V. Daftardar-Gejji, "Solving a system of nonlinear fractional differential equations using Adomian decomposition," Journal of Computational and Applied Mathematics, vol. 196, no. 2, pp. 644-651, 2006.

[18] D. Lesnic, "The decomposition method for Cauchy advectiondiffusion problems," Computers \& Mathematics with Applications, vol. 49, no. 4, pp. 525-537, 2005.

[19] D. Lesnic, "The decomposition method for initial value problems," Applied Mathematics and Computation, vol. 181, no. 1, pp. 206-213, 2006.

[20] V. Daftardar-Gejji and H. Jafari, "Adomian decomposition: a tool for solving a system of fractional differential equations," Journal of Mathematical Analysis and Applications, vol. 301, no. 2, pp. 508-518, 2005.

[21] Z. M. Odibat and S. Momani, "Application of variational iteration method to nonlinear differential equations of fractional order," International Journal of Nonlinear Sciences and Numerical Simulation, vol. 7, no. 1, pp. 27-34, 2006.

[22] S. Momani and Z. Odibat, "Analytical approach to linear fractional partial differential equations arising in fluid mechanics," Physics Letters A, vol. 355, no. 4-5, pp. 271-279, 2006.

[23] V. Daftardar-Gejji and H. Jafari, "An iterative method for solving nonlinear functional equations," Journal of Mathematical Analysis and Applications, vol. 316, no. 2, pp. 753-763, 2006.

[24] M. Zurigat, S. Momani, Z. Odibat, and A. Alawneh, "The homotopy analysis method for handling systems of fractional differential equations," Applied Mathematical Modelling, vol. 34, no. 1, pp. 24-35, 2010.

[25] V. S. Ertürk and S. Momani, "Solving systems of fractional differential equations using differential transform method," Journal of Computational and Applied Mathematics, vol. 215, no. 1, pp. 142-151, 2008.

[26] M. Alipour, D. Rostamy, and D. Baleanu, "Solving multidimensional FOCPs with inequality constraint by BPs operational matrices," Journal of Vibration and Control, 2012.

[27] D. Rostamy and K. Karimi, "Bernstein polynomials for solving fractional heat- and wave-like equations," Fractional Calculus and Applied Analysis, vol. 15, no. 4, pp. 556-571, 2012.

[28] D. Rostamy, M. Alipour, H. Jafari, and D. Baleanu, "Solving multi-term orders fractional differential equations by operational matrices of BPs with convergence analysis," Romanian Reports in Physics, vol. 65, no. 2, 2013.

[29] S. Momani, A. Abu Rqayiq, and D. Baleanu, "A nonstandard finite difference scheme for two-sided space-fractional partial differential equations," International Journal of Bifurcation and Chaos in Applied Sciences and Engineering, vol. 22, no. 4, Article ID 1250079, 2012.

[30] E. W. Cheney, Introduction to Approximation Theory, AMS Chelsea Publishing, Providence, RI, USA, 2nd edition, 1982.

[31] E. Kreyszig, Introduction Functional Analysis with Applications, John Wiley \& Sons, New York, NY, USA, 1978.

[32] M. Alipour and D. Rostamy, "Bernstein polynomials for solving Abel's integral equation," The Journal of Mathematics and Computer Science, vol. 3, no. 4, pp. 403-412, 2011.

[33] C. Canuto, M. Y. Hussaini, A. Quarteroni, and T. A. Zang, Spectral Methods in Fluid Dynamic, Prentice-Hall, Englewood Cliffs, NJ, USA, 1988. 


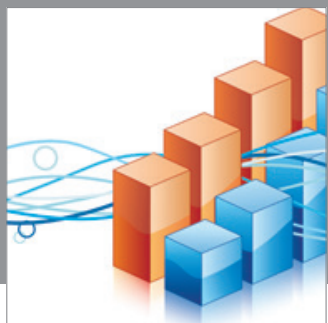

Advances in

Operations Research

mansans

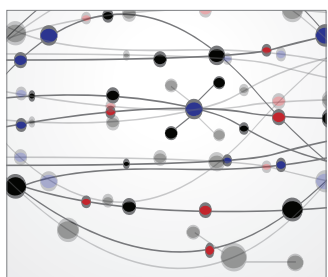

The Scientific World Journal
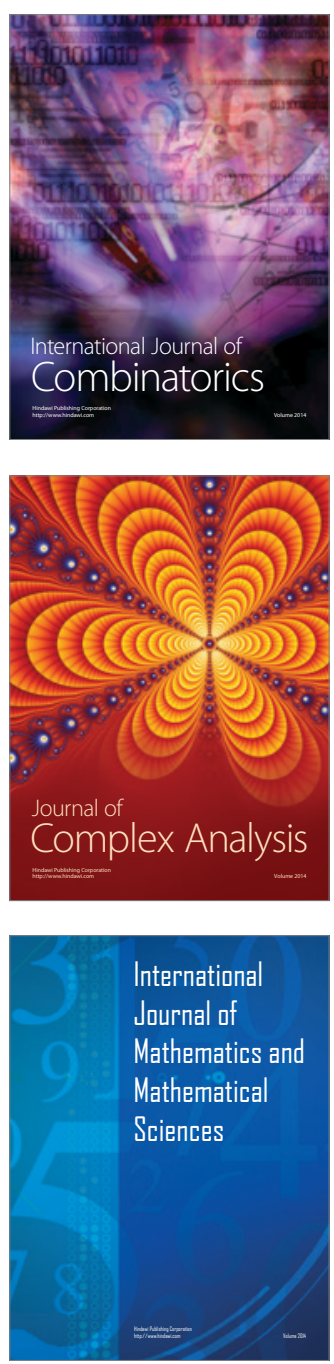
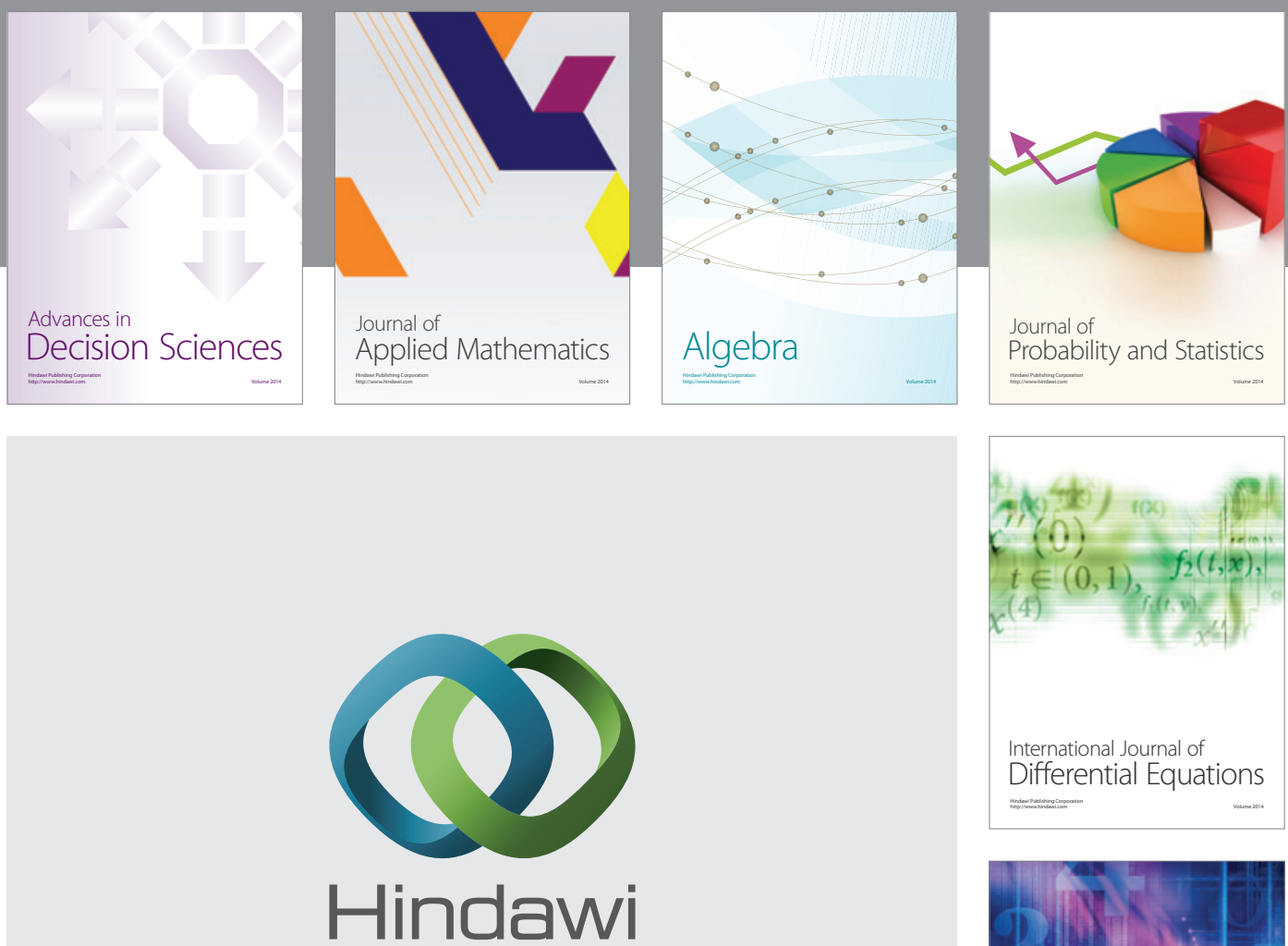

Submit your manuscripts at http://www.hindawi.com
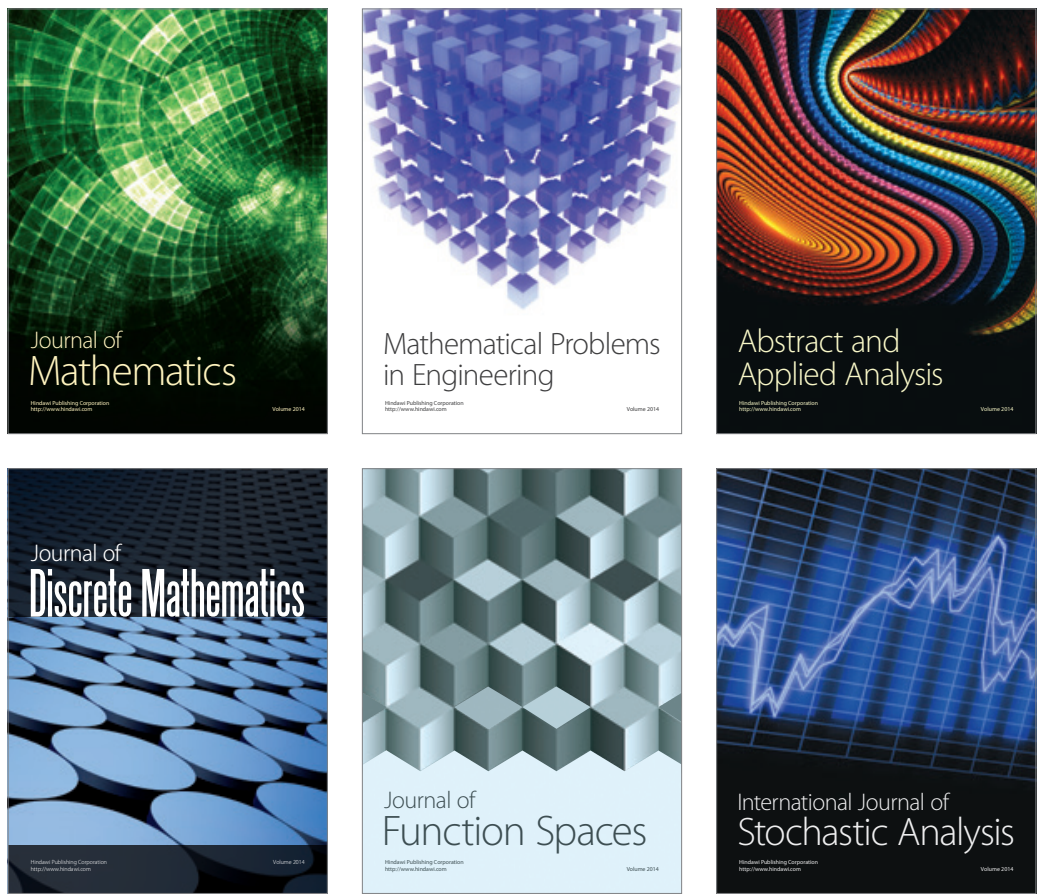

Journal of

Function Spaces

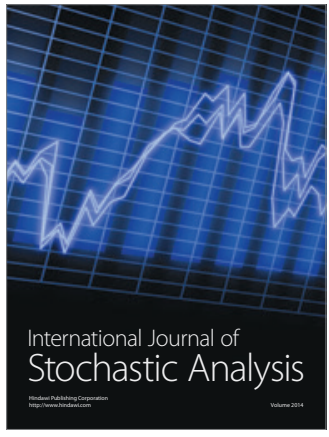

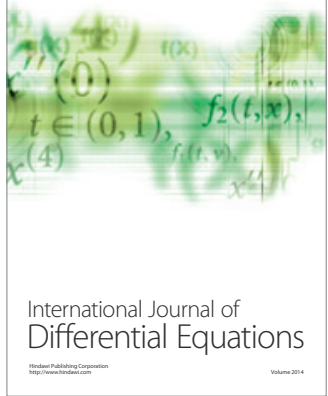
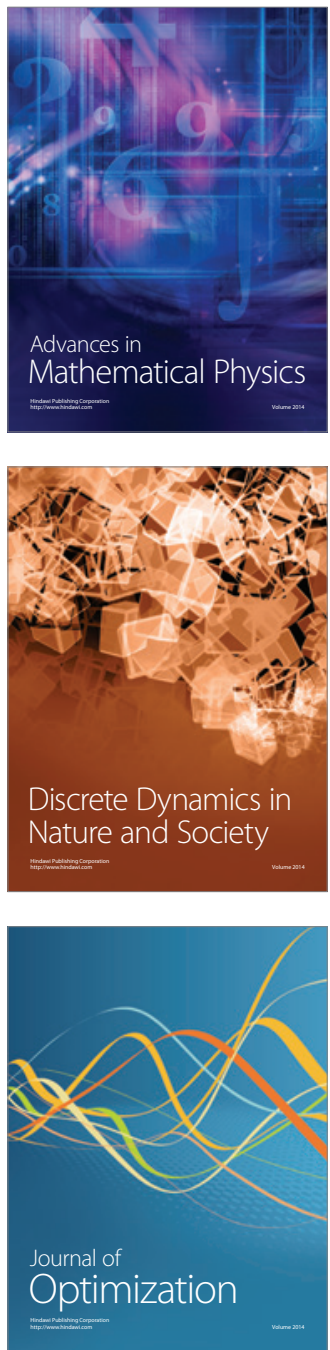